\title{
Air lens vs aspheric surface: a lens design case study
}

\section{Weichuan Gao, José Sasián}

Weichuan Gao, José Sasián, "Air lens vs aspheric surface: a lens design case study," Proc. SPIE 10590, International Optical Design Conference 2017, 105900B (27 November 2017); doi: 10.1117/12.2287888

SPIE Event: International Optical Design Conference - IODC 2017, 2017, Denver, United States 


\title{
Air Lens vs Aspheric Surface: A Lens Design Case Study \\ Weichuan Gao*, José Sasián \\ College of Optical Sciences, University of Arizona, Tucson, AZ, 85721
}

\begin{abstract}
We discuss the behavior of air lenses in lens design. The structural aberration coefficients of a thin air lens are derived and compared with their glass thin lens counterpart. Examples are provided for a telephoto lens and the Monochromatic Quartet where air lenses or aspheric surfaces are used.
\end{abstract}

Keywords: Air lenses, aspheric surfaces, telephoto lens, monochromatic quartet, structural coefficients

\section{INTRODUCTION}

The concept of air lens was developed since the early days of lens design. They are recognized in the development of the Zeiss Unar lens and the Dallmeyer's Stigmatic lens. By separating a cemented component to create an air lens an additional degree of freedom is added to fulfill the anastigmatic condition [1]. In many ways, the properties of an air lens resemble that of a refractive lens. However, sometimes air lenses can provide unique properties or insights in the lens design process. An earlier discussion by D. Shafer focused on air lenses and their glass equivalents for generating new design forms [2]. For example, Shafer showed that the astigmatism correction of a compact doublet and the shortened version of a Houghton system were made possible from the fact that the glass equivalent of a nearly concentric air lens acts as a remote element.

In the present paper, we first provide the structural aberration coefficients of an air lens. Second, we provide a case study of a telephoto where an air lens results in a more relaxed, better performing lens design form. We also show that in this case the air lens is superior to using an aspheric surface and thus shows that aspheric surfaces have disadvantages beyond manufacturing. Finally, the design of the monochromatic quartet is discussed from the principles of aberration theory. Observations from the quartet design lead to some equivalent triplet designs using aspheric surfaces.

\section{STRUCTURAL ABERRATION COEFFICIENTS OF A THIN AIR LENS}

Structural aberration coefficients have been used in lens design for setting up starting points and for assessing system trade-offs [3]. Here we use structural aberration coefficients to study the behavior of a thin air lens. To simplify the formulas, refractive indices on both sides of the air lens are made the same. As shown in Table 1, the structural aberration coefficients for the five primary aberrations with stop at the air lens are compared with a refractive thin lens in

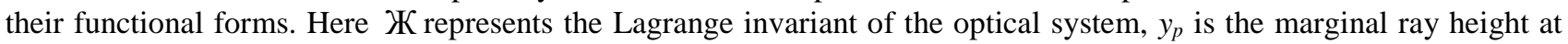
the principal planes, and $\Phi$ is the optical power of the thin lens with spherical surfaces.

Table 1. Structural aberration coefficients of a thin air lens with refractive index $n$ on both sides.

\begin{tabular}{|l|l|l|}
\hline $\begin{array}{c}\text { Seidel sums in terms of } \\
\text { structural aberration coefficients }\end{array}$ & $\begin{array}{c}\text { Structural aberration } \\
\text { coefficients of a thin glass lens }\end{array}$ & $\begin{array}{c}\text { Structural aberration } \\
\text { coefficients of a thin air lens }\end{array}$ \\
\hline$S_{I}=\frac{1}{4} y_{P}^{4} \Phi^{3} \sigma_{I}$ & $\sigma_{I}=A X^{2}-B X Y+C Y^{2}+D+\alpha$ & $\sigma_{I}=A X^{2}+B X Y+C Y^{2}+D+\alpha$ \\
\hline$S_{I I}=\frac{1}{2} Ж y_{P}^{2} \Phi^{2} \sigma_{I I}$ & $\sigma_{I I}=E X-F Y$ & $\sigma_{I I}=-E X-F Y$ \\
\hline$S_{I I I}=Ж^{2} \Phi \sigma_{I I I}$ & $\sigma_{I I}=1$ & $\sigma_{I I I}=\frac{1}{n^{2}}$ \\
\hline$S_{I V}=Ж^{2} \Phi \sigma_{I V}$ & $\sigma_{I V}=\frac{1}{n}$ & $\sigma_{I V}=\frac{1}{n}$ \\
\hline
\end{tabular}

International Optical Design Conference 2017, edited by Peter P. Clark, Julius A. Muschaweck, Richard N. Pfisterer, John R. Rogers, Proc. of SPIE-OSA Vol. 10590, 105900B - @ 2017 SPIE CCC code: $0277-786 X / 17 / \$ 18 \cdot$ doi: $10.1117 / 12.2287888$ 


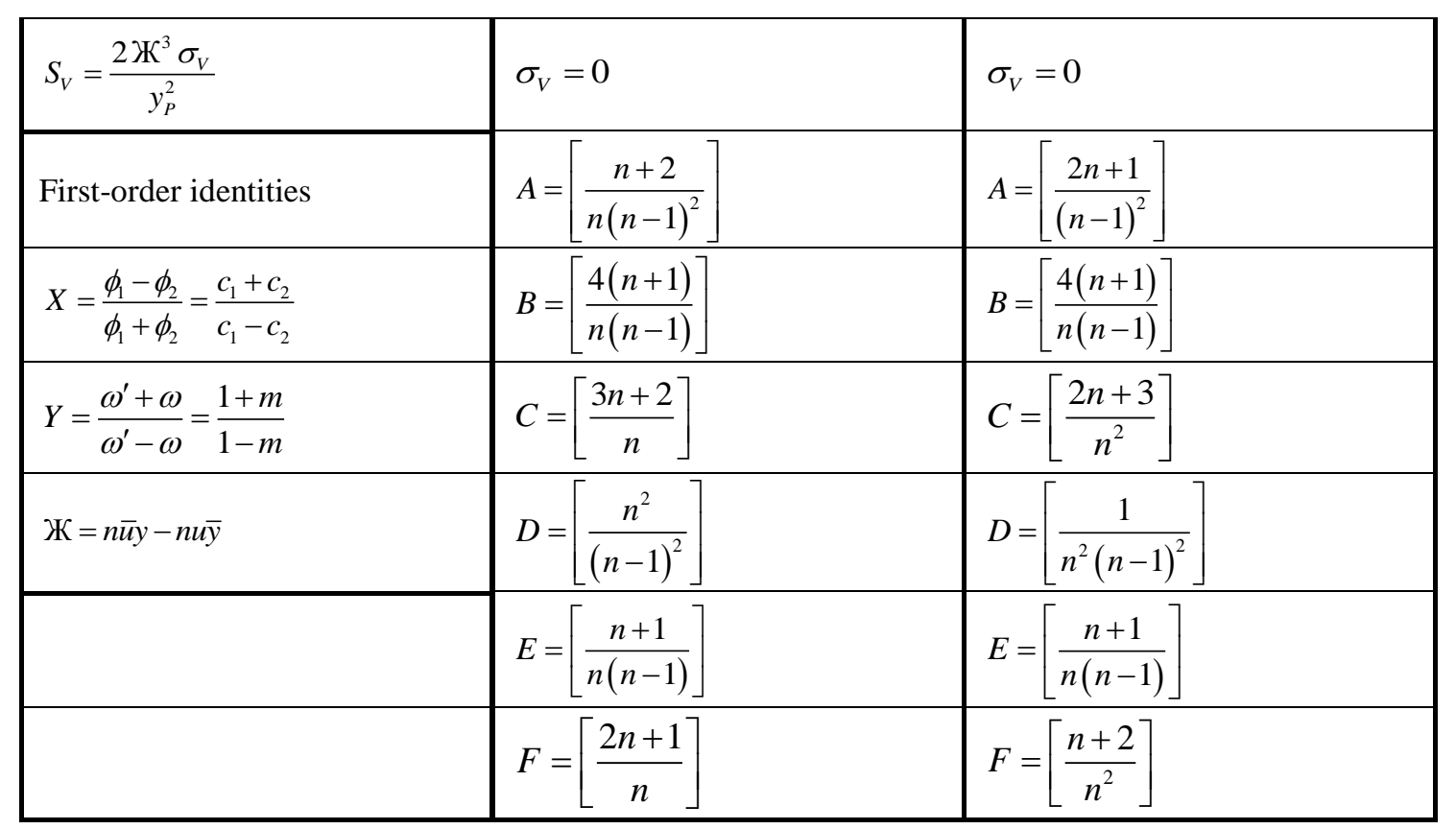

The signs before the coefficients $B$ and $E$ change between the glass lens and the air lens. This change of sign is merely a result of the definition of the shape factor $X$. For instance, a plano-concave air lens with $X=-1$ is functionally equivalent to a convex-plano refractive lens with $X=1$ instead of a plano-convex refractive lens with $X=-1$. Compared with the glass lens, the astigmatism introduced by a thin air lens decreases by a factor of $n^{2}$. This may have a large impact in the aberration correction as the material refractive index increases. As shown in Table 2, coefficients A through $\mathrm{F}$ for different indexes are compared. The spherical aberration and coma introduced by the glass lens and the air lens also shows significantly different dependence on the material index of refraction.

Table 2. A, B, C, D, E, F coefficients for a glass lens and an air lens with different material refractive indexes

\begin{tabular}{|c|c|c|c|c|c|c|}
\hline & \multicolumn{2}{|c|}{$\mathrm{n}=1.5$} & \multicolumn{2}{c|}{$\mathrm{n}=2$} & \multicolumn{2}{c|}{$\mathrm{n}=4$} \\
\hline & glass lens & air lens & glass lens & air lens & glass lens & air lens \\
\hline A & 9.33 & 16 & 2 & 5 & 0.17 & 1 \\
\hline B & 13.33 & 13.33 & 6 & 6 & 1.67 & 1.67 \\
\hline C & 4.33 & 2.67 & 4 & 1.75 & 3.5 & 0.69 \\
\hline D & 9 & 1.78 & 4 & 0.25 & 1.78 & 0.007 \\
\hline E & 3.33 & 3.33 & 1.5 & 1.5 & 0.42 & 0.42 \\
\hline F & 2.67 & 1.56 & 2.5 & 1 & 2.25 & 0.38 \\
\hline
\end{tabular}

\section{DISTORTION CORRECTION IN A TELEPHOTO LENS}

Telephoto lenses consist of a positive component followed by a negative component with the stop aperture in between. Telephoto lenses are known to have some degree of pincushion distortion. H. Lee first tackled the correction of distortion by separating the rear component and creating a collective air lens $[4,5]$. A similar telephoto lens has been studied showing that only certain configuration of the rear air-spaced doublet allow correction for distortion [6].

To illustrate the correction of distortion in view of the air lens concept we have set up several telephoto lenses with $200 \mathrm{~mm}$ focal length, F/4, 0.8 telephoto ratio and stop at the front positive component. Design layouts and performance merits with rear negative components being cemented doublets (Design 1 and 2), air-spaced doublets (Design 3 and 4) 
and cemented doublet with an aspheric surface (highlighted in Design 5) are shown in Table 3. Though, all the latter 3 designs can achieve the correction of distortion aberration, both the designs with the positive air lens and aspheric surface show slightly degraded performance across the field of view.

Table 3. Telephoto lenses with $\mathrm{f}=200 \mathrm{~mm}, \mathrm{~F} / 4$ and telephoto ratio $=0.8$

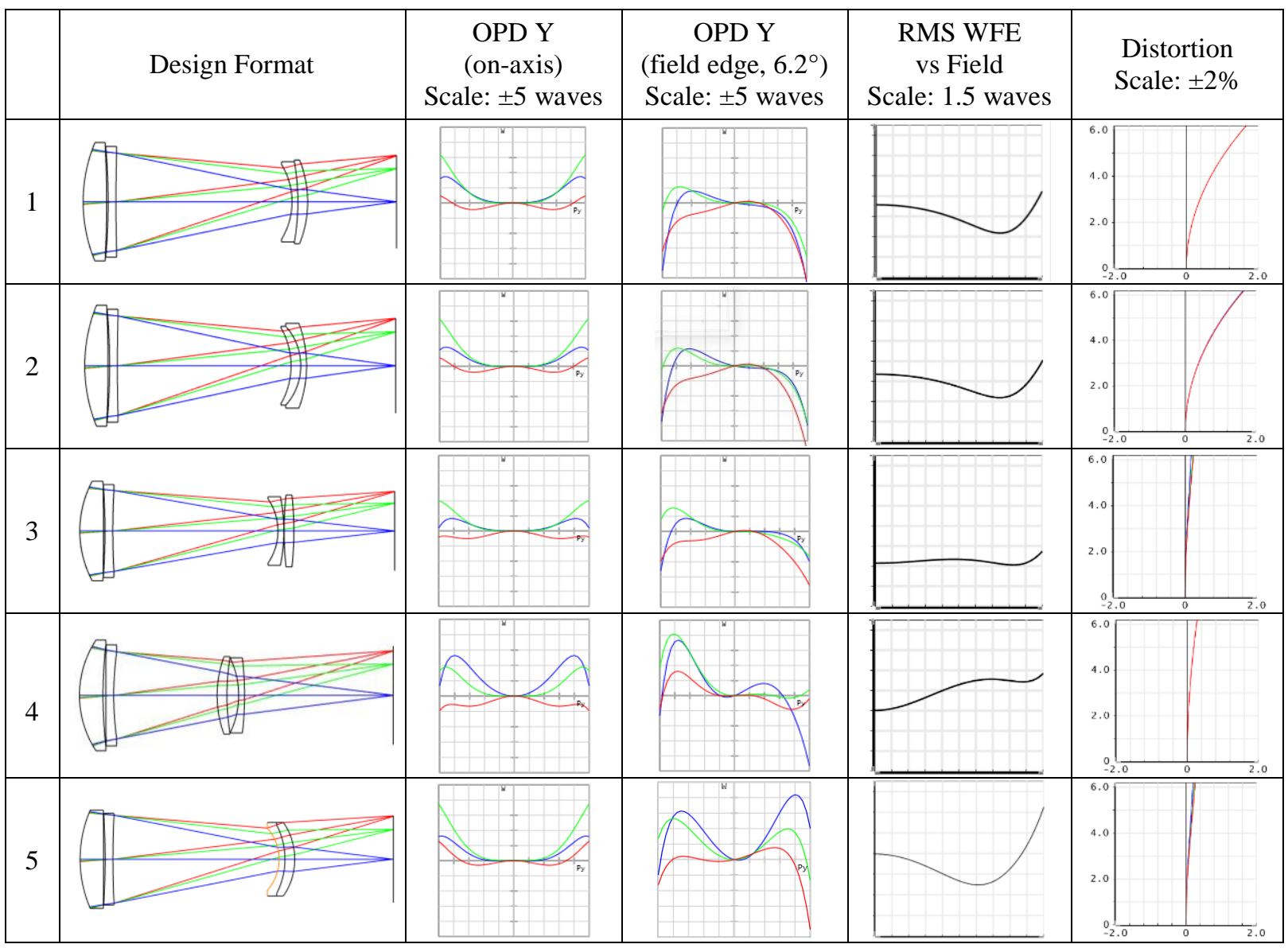

With the stop aperture at the front positive doublet, the distortion and astigmatism contribution are fixed as they do not depend on lens shape. For an air-spaced front doublet, two degrees of freedom are used to correct for spherical and coma aberrations. While the two degrees of freedom of the rear doublet are used to correct for distortion and astigmatism aberrations. In Design 4 with the positive air lens, the rear doublet moves towards the front which changes the power contributed by the rear doublet and makes the positive air lens to increase its optical power. Thus, the front doublet must generate more spherical and coma aberrations to cancel the aberrations from the positive air lens. The result is a more stressed form of design with the increase of higher order aberrations.

Alternative insights can be obtained by analyzing the power distribution parameter $W$ and the symmetry parameter $S$ of the systems [7]. In Table 4, Design 4 has a large $W$ indicating the inefficient power distribution among optical surfaces. Such a system tends to generate large amounts of aberrations to cancel against large amounts and thus is prone to generating higher order aberration residuals. In Design 5 that has a large $S$ parameter, the aspheric surface is used for controlling astigmatism. Generally, systems with smaller $W$ and $S$ will have more imaging potential. In this case, Design 3 is the best performer as suggested by the overall lower power distribution and symmetry parameters.

Table 4. Power distribution and symmetry parameters of the telephoto designs

\begin{tabular}{|c|c|c|c|c|c|}
\hline & Design 1 & Design 2 & Design 3 & Design 4 & Design 5 \\
\hline $\mathrm{W}$ & 1.014 & 1.007 & 1.005 & 1.465 & 1.246 \\
\hline $\mathrm{S}$ & 1.452 & 1.418 & 1.360 & 1.283 & 2.007 \\
\hline
\end{tabular}




\section{MONOCHROMATIC QUARTET CORRECTION AND ALTERNATE DESIGNS}

The Monochromatic Quartet design from the 1990 International Lens Design Conference is a problem seeking out the best four-element monochromatic design for an imaging lens with a moderate field of $30^{\circ}$ at a moderate speed of $\mathrm{f} / 3$ [8]. Shown in Figure 1 are two of the best entries that share a similar construction with the only exception in the shape of the $1^{\text {st }}$ air lens. These solutions provide very good imaging quality monochromatically with low distortion. It is shown that the best design form is most likely to be associated with a relaxed field behavior and its large volume filled with glass is again an indication of the positive influence of lens overall size on image quality. [9]
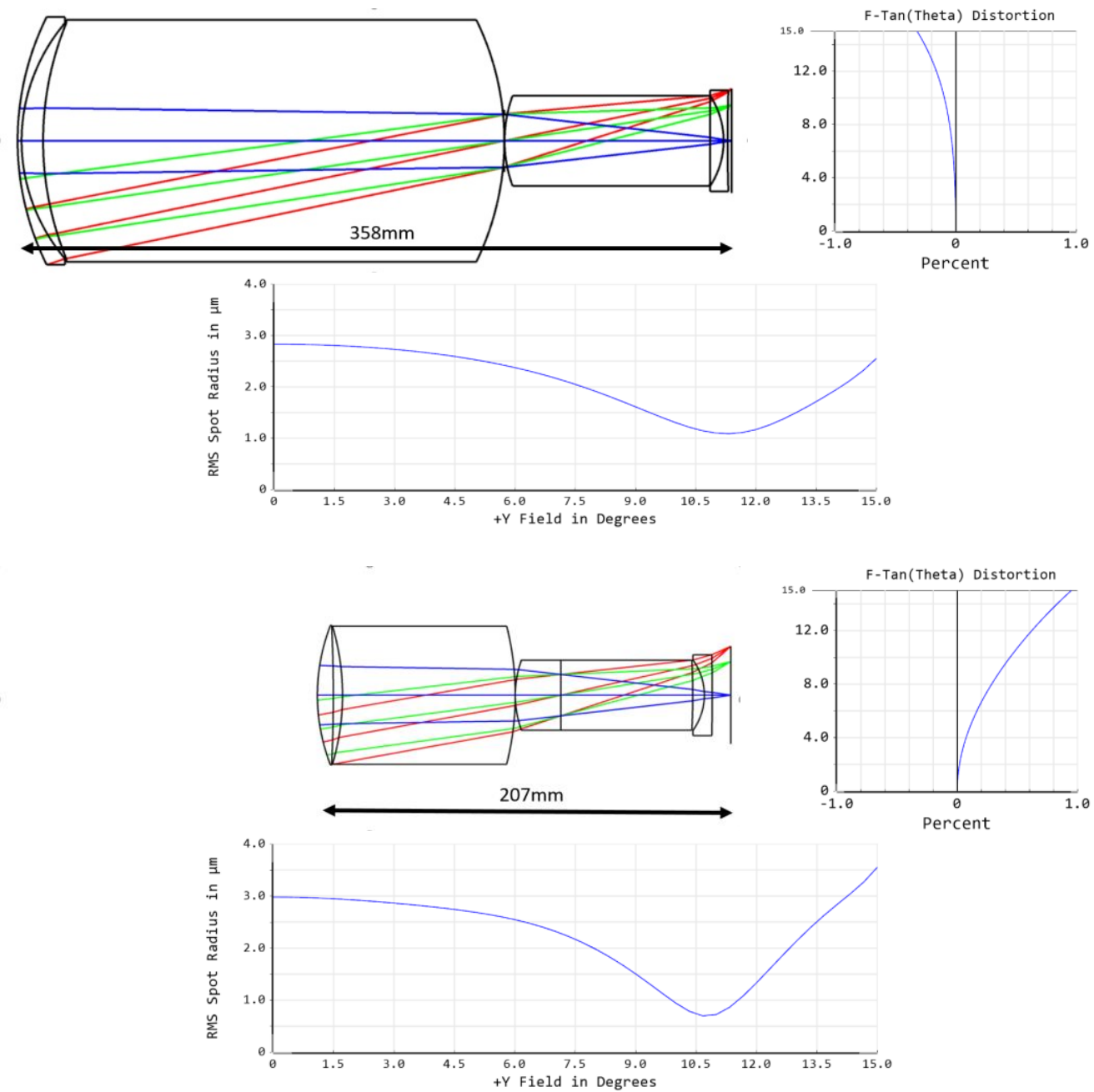

Fig. 1. Optical layouts and performance of the two best entries for the Monochromatic Quartet problem.

One interesting observation from these two best designs is that the first 3 surfaces contribute little optical power to the overall system power. Also the last air lens close to the image plane has little contribution to the overall optical power of the system. By replacing the first 3 surfaces with a flat (no curvature on-axis) aspheric surface, the Monochromatic Quartet becomes a triplet with all the optical power provided by the air lens located at the aperture stop. The roles of the 3 separated groups then become obvious. The two degrees of freedom of the flat aspheric surface (location and asphericity) are used to correct spherical aberration and astigmatism, the one degree of freedom of the air lens at the stop (bending) is used to correct coma aberration, and the two degrees of freedom of the last air lens near the image (bending and power) are used to correct Petzval field curvature and distortion aberrations. A $3^{\text {rd }}$ order solution can be analytically solved and is shown in Figure 2 with a $n_{d}=1.5$ model glass material. 


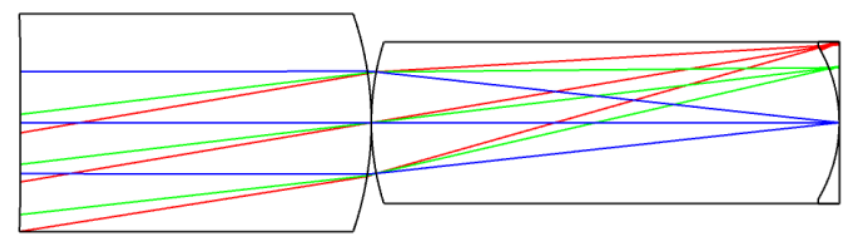

\begin{tabular}{|c|c|c|c|c|c|c|c|c|c|c|}
\hline \multicolumn{3}{|c|}{ Surf:Type } & \multirow[t]{2}{*}{ Comment } & \multirow{2}{*}{$\begin{array}{l}\text { Radius } \\
\text { Infinity }\end{array}$} & \multirow{2}{*}{$\begin{array}{r}\text { Thickness } \\
\text { Infinity }\end{array}$} & \multirow[t]{2}{*}{ Material } & \multirow{2}{*}{$\begin{array}{c}\text { Semi-Diamete } \\
\text { Infinity }\end{array}$} & \multirow{2}{*}{$\begin{array}{c}\text { Conic } \\
0.00000\end{array}$} & \multirow[t]{2}{*}{ 4th Order Term } & 6th Order Ter \\
\hline 0 & OBJECT & Standard $\mathbf{v}$ & & & & & & & & \\
\hline 1 & & Even Asphere & & Infinity & 112.50000 & $1.50,0.0$ & 35.76115 & 0.00000 & $-1.97531 E-007$ & 0.00000 \\
\hline 2 & & Standard $\mathbf{V}$ & & -112.50000 & 0.00000 & & 17.68357 & 0.00000 & & \\
\hline 3 & STOP & Standard & & 90.00000 & $150.00000 \mathrm{M}$ & $1.50,0.0$ & 16.66666 & 0.00000 & & \\
\hline 4 & & Standard $\mathbf{V}$ & & -750.00000 & 0.00000 & & 26.34145 & 0.00000 & & \\
\hline 5 & & Standard - & & -46.87500 & 0.00000 & $1.50,0.0$ & 25.05917 & 0.00000 & & \\
\hline 6 & & Standard $\mathbf{V}$ & & Infinity & 0.00000 & & 26.86854 & 0.00000 & & \\
\hline 7 & IMAGE & Standard $\mathbf{v}$ & & Infinity & - & & 26.86854 & 0.00000 & & \\
\hline
\end{tabular}

Seidel Aberration Coefficients:

\begin{tabular}{|c|c|c|c|c|c|}
\hline Surf & SPHA & COMA & ASTI & FCUR & DIST \\
\hline 1 & -0.060966 & 0.073511 & -0.088638 & 0.000000 & 0.285006 \\
\hline 2 & 0.060966 & -0.073511 & 0.088638 & 0.059092 & -0.178129 \\
\hline STO & -0.000000 & -0.000000 & -0.000000 & 0.073865 & 0.178129 \\
\hline 4 & 0.000000 & -0.000000 & 0.000000 & 0.008864 & -0.011400 \\
\hline 5 & -0.000000 & -0.000000 & -0.000000 & -0.141821 & $-0 \mid .273605$ \\
\hline 6 & 0.000000 & -0.000000 & 0.000000 & -0.000000 & 0.000000 \\
\hline IMA & 0.000000 & 0.000000 & 0.000000 & 0.000000 & 0.000000 \\
\hline ТОТ & -0.000000 & -0.000000 & -0.000000 & 0.000000 & -0.000000 \\
\hline
\end{tabular}
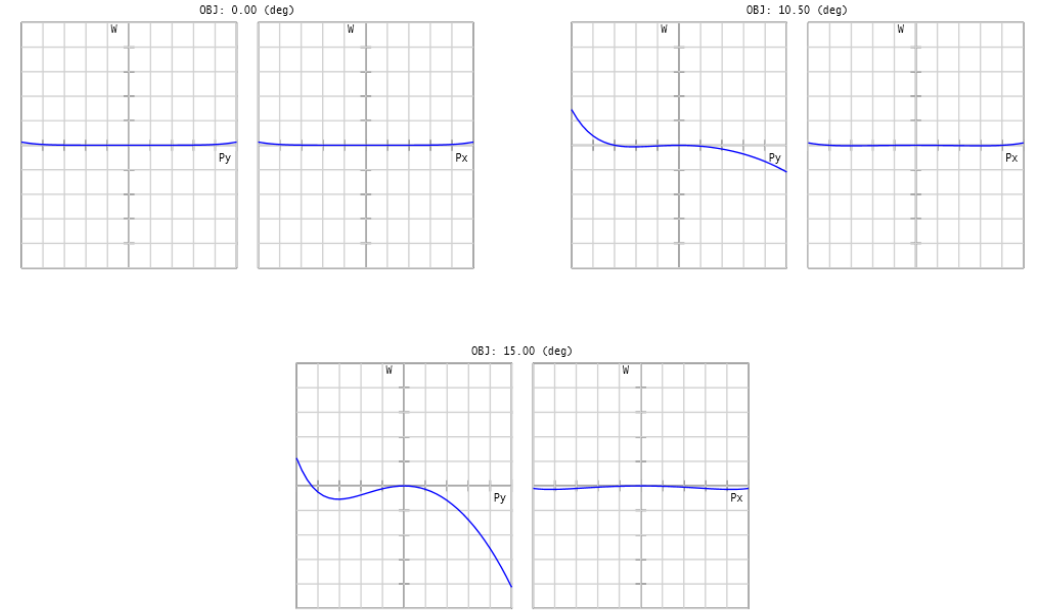

Fig. 2. $3^{\text {rd }}$ order solution for the triplet with a flat aspheric surface and two air lenses. Optical layout and lens data (top), Seidel aberration coefficients of the triplet (middle), OPD plots at on-axis, +0.7 and +1 field (bottom)

As seen in the OPD plots, the image quality of this $3^{\text {rd }}$ order solution is limited by higher order coma and astigmatism aberrations. These higher order aberrations result from where large opposite aberrations are balanced between the flat aspheric surface and the air lens at stop. Through calculations, it becomes clear that a unique $3^{\text {rd }}$ order solution exists regardless of the curvature of the $1^{\text {st }}$ aspheric surface. Thus, all radii of the triplet can be made variables for optimization. In addition, the $6^{\text {th }}$ order aspheric coefficient on the $1^{\text {st }}$ surface can be used for the correction of higher order field aberrations. An optimized design is shown in Figure 3. Compared with the best quartet designs, this aspheric triplet design provides similar image quality. Since for any power distribution between the $1^{\text {st }}$ surface and the air lens at the stop, there is a unique solution of the $3^{\text {rd }}$ order. The search for the best power distribution is indeed an optimization for minimum residual higher order field aberrations. 

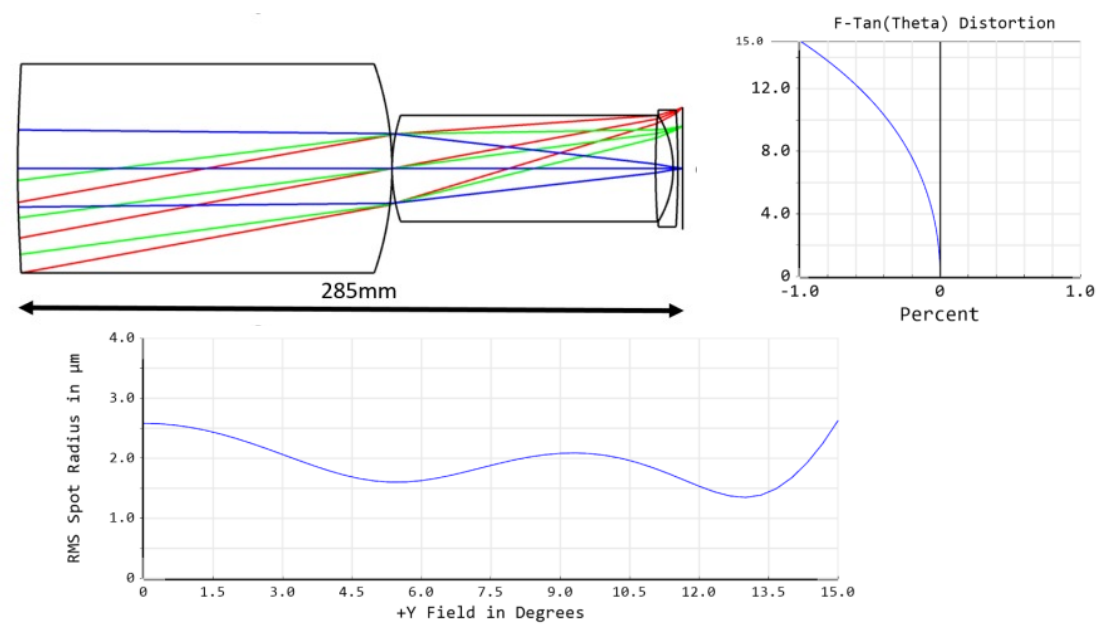

Fig. 3. Optical layout and performance of the aspheric triplet.

As shown in Figure 1, two distinct solutions exist in the design space of the Monochromatic Quartet. The 1st solution has the 1 st air lens with its shape factor $\mathrm{X}$ being positive, while for the 2 nd solution, the shape factor for the 1 st air lens is close to -1. By assessing a 3rd order solution with structural aberration coefficients, it soon becomes clear that the two setups of the air lenses introduce similar amounts of spherical aberration into the system. This is the solutions are the result from the quadratic dependence of spherical aberration on the shape factor $\mathrm{X}$.

There is also a thin lens triplet solution associated with this configuration that provides comparable image quality. As shown in Figure 4, each of the air lenses are replaced with their thin glass lens counterparts. Note in this design, aspheric surfaces are required on both the 1 st and 2 nd elements to achieve a comparable image quality with the Monochromatic Quartet. The asphericity at the 1st element provides a similar function as in the previous design for the correction of higher order coma and astigmatism aberrations. The aspheric surface at the 2 nd element becomes necessary as an additional degree of freedom for correction of spherical aberration when the air lens is replaced by a glass lens.

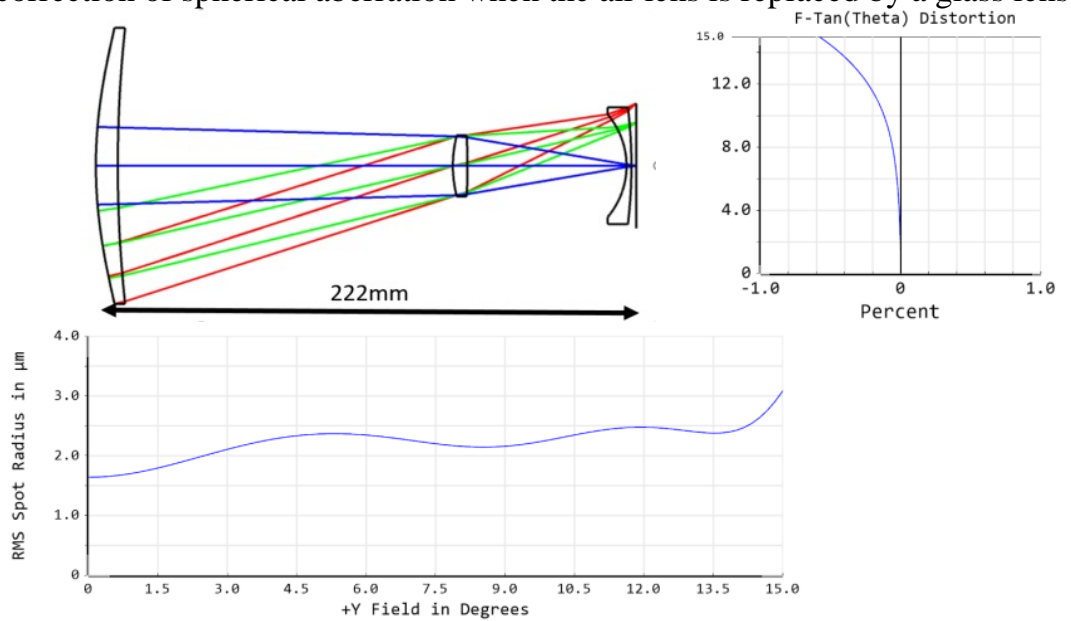

Fig. 4. Optical layout and performance of the aspheric glass lens triplet.

\section{CONCLUSIONS}

While lens splitting and adding an aspheric surface are sometimes seen as equivalent degrees of freedom, their behavior can be certainly different as the structural aberration coefficients of air lenses and aspheric surfaces behave differently. In the telephoto case study, we showed that an air lens may offer unique properties in the correction of aberration. An aspheric surface is not in general equivalent to lens splitting. Further, introducing air lenses, which is commonly referred to as lens splitting, does not guarantee a more relaxed design form. For the Monochromatic Quartet we showed that under certain circumstances air lenses and aspheric surfaces can be equivalent to each other. However, we also show that it is not unusual to require adding a degree of freedom (asphericity for example) when an air lens is replaced by its glass counterpart. 


\section{REFERENCES}

[1] Lummer, O., [Contributions to Photographic Optics]. London: Macmillan and co., 1900.

[2] Shafer, D., "Optical Design with Air Lenses," Proc. SPIE 0399, Optical System Design, Analysis, and Production, 186 (1983).

[3] Sasián, J., [Introduction to aberrations in optical imaging systems]. Cambridge University Press, 2012.

[4] Taylor,W. and Lee. H.W., "The development of the photographic lens," Proceedings of the Physical Society, 47.3 (1935).

[5] Kingslake, R., "Telephoto vs. Ordinary Lenses: A Tutorial Paper," in Journal of the SMPTE, vol. 75, no. 12, pp. 1165-1168, (1966).

[6] Kazamaki, T. and Kondo, F., "New Series of Distortionless Telephoto Lenses," J. Opt. Soc. Am. 46, 22-31 (1956).

[7] Sasian, J., Descour, M., "Power distribution and symmetry in lens systems," Optical Engineering, 0001; 37(3):10011004 (1998).

[8] O’Shea, D. C. "The monochromatic quartet: A search for the global optimum." Proc. SPIE, Vol. 1354. (1990).

[9] Williamson, D. M., "The monochromatic quartet explained," in Lens Design, Warren Smith, Ed., SPIE Press, Bellingham, WA (1992). 